\title{
Electroencephalogram Domain
}

National Cancer Institute

\section{Source}

National Cancer Institute. Electroencephalogram Domain. NCI Thesaurus. Code C49625.

A subject domain utilized for the submission of information encompassing and representing data, vocabulary or records related to electroencephalogram. 